\title{
A patient with chest pain and a pulsating left main coronary artery
}

\author{
A. Y. Andreou · A. Karyou · A. Argyrou
}

Published online: 14 August 2019

(C) The Author(s) 2019

A 42-year-old female patient, a cigarette smoker with no known medical history, was referred for emergency coronary angiography because of acute coronary syndrome (ACS). She presented with suddenonset chest pain associated with electrocardiographic evidence of ischaemia (Fig. 1a). Her blood pressure was $90 / 25 \mathrm{mmHg}$. The right radial artery pulse was non-palpable. Thus we performed transfemoral coronary angiography, which showed no evidence of atherosclerosis but a smooth-bordered ostial and mid-shaft left main coronary artery (LMCA) stenosis with dynamic compression and almost complete lumen obliteration during diastole (Fig. 1b, c; Electronic Supplementary Material, Video 1). We suspected proximal aortic dissection (AD), which was confirmed by emergency echocardiography (Electronic Supplementary Material, Video 2). Indeed, LMCA pulsation was due to retrograde extension of the aortic false lumen into the LMCA causing diastolic compression of the true coronary lumen. The patient underwent a successful $\mathrm{AD}$ repair with Bentall's procedure and hemi-arch replacement and patch repair of the dissected LMCA.

Electronic supplementary material The online version of this article (https://doi.org/10.1007/s12471-019-01324-w) contains supplementary material, which is available to authorized users.

\footnotetext{
A. Y. Andreou $(\bowtie) \cdot$ A. Karyou

Department of Cardiology, Limassol General Hospital,

Limassol, Cyprus

y.andreas@yahoo.com
}

A. Y. Andreou

University of Nicosia Medical School, Nicosia, Cyprus

\section{A. Argyrou}

Department of Cardiology, Paphos General Hospital,

Paphos, Cyprus
Acute proximal $\mathrm{AD}$ is complicated by retrograde dissection into either or both coronary ostia in $5.7-15 \%$ of cases [1, 2]; misdiagnosis with ACS may have a fatal outcome [3]. Dynamic LMCA lumen compromise during diastole is highlighted herein as a subtle and rare angiographic finding that should alert the interventional cardiologist to possible proximal $\mathrm{AD}$ extending into the LMCA.

Conflict of interest A.Y. Andreou, A. Karyou and A. Argyrou declare that they have no competing interests.

Open Access This article is distributed under the terms of the Creative Commons Attribution 4.0 International License (http://creativecommons.org/licenses/by/4.0/), which permits unrestricted use, distribution, and reproduction in any medium, provided you give appropriate credit to the original author(s) and the source, provide a link to the Creative Commons license, and indicate if changes were made.

\section{References}

1. NeriE, ToscanoT, Papalia U, et al. Proximalaortic dissection with coronary malperfusion: presentation, management, and outcome. J Thorac Cardiovasc Surg. 2001;121:552-60.

2. Imoto $\mathrm{K}$, Uchida $\mathrm{K}$, Karube N, et al. Risk analysis and improvement of strategies in patients who have acute type A aortic dissection with coronary artery dissection. Eur J Cardiothorac Surg. 2013;44:419-24.

3. Kawano H, Tomichi Y, Fukae S, Koide Y, Toda G, Yano K. Aortic dissection associated with acute myocardial infarction and strokefound at autopsy. Intern Med. 2006;45:957-62. 
Fig. 1 a Twelve-lead electrocardiogram on admission depicting ST-segment depression and negative $\mathrm{T}$ wave in leads II, aVF, III and V2-V6 and STsegment elevation in lead aVR. b Non-selective left coronary artery (LCA) angiogram (systolic frame) in the left anterior oblique (LAO) caudal projection depicting a narrowed left main coronary artery (LMCA) due to an obstructing false lumen that extended from the aorta. c Non-selective LCA angiogram in the LAO caudal projection depicting a slit-like lumen of the LMCA in diastole due to compression by the false lumen
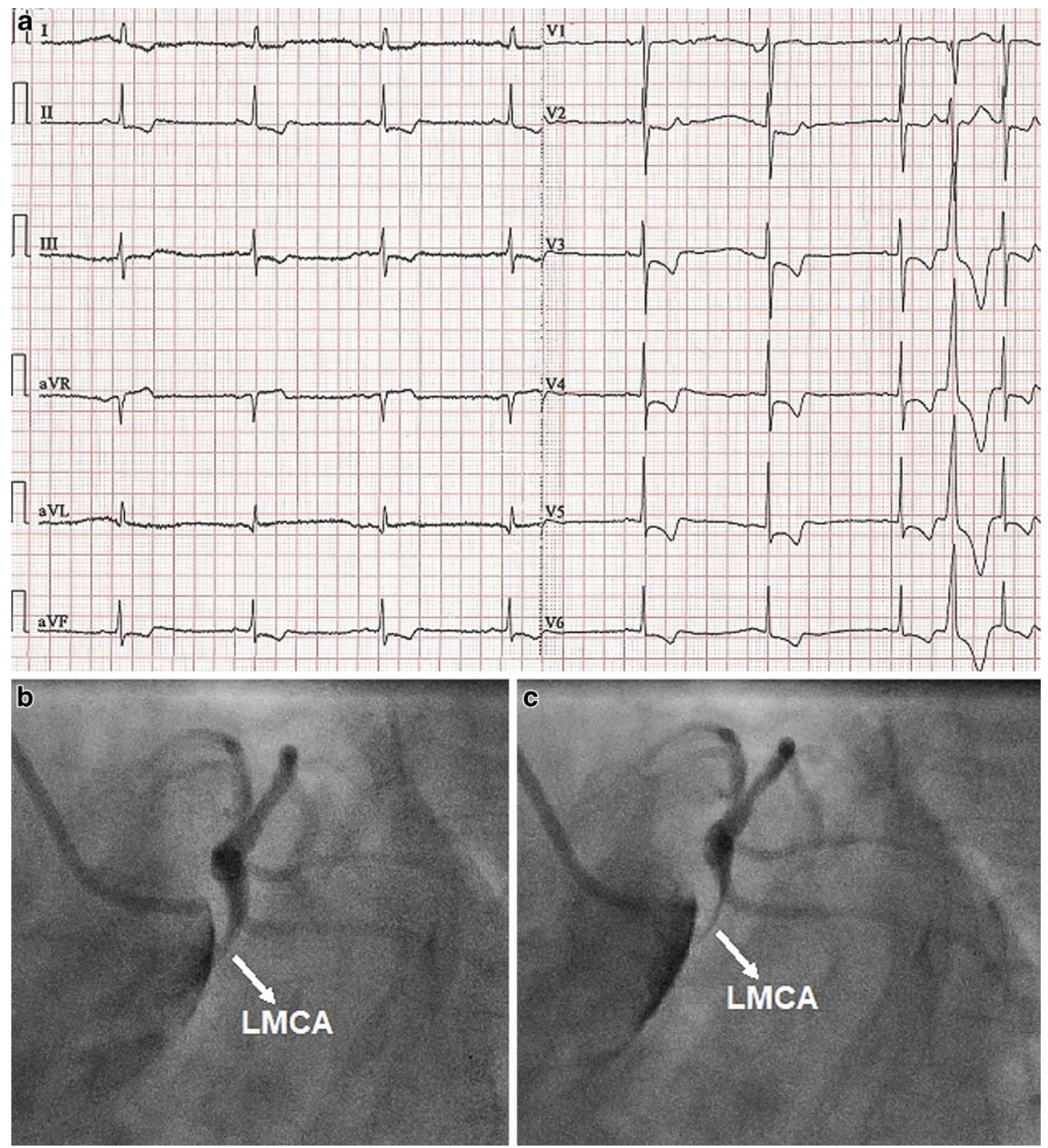